\title{
DYNAMIQUE TEMPORELLE DE LA CONTRIBUTION DU REPEUPLEMENT EN STADES PRÉCOCES DE TRUITE (SALMO TRUTTA L.) DANS LA POPULATION EN PLACE ET DANS LA PÊCHE SUR LE DOUBS FRANCO-SUISSE.
}

\author{
A. CHAMPIGNEULLE (1), F. DEGIORGI (2), J.C. RAYMOND (3), S. CACHERA (4)
}

(1) Station d'Hydrobiologie Lacustre, INRA, BP 511, 74203 THONON Cedex, France.

(2) TELEOS, Château de la Bouloie, 20 route de Gray, 25000 BESANCON, France.

(3) Conseil Supérieur de la Pêche, Délégation Régionale 5, Parc de Parilly, Chemin des Chasseurs, 69500 BRON, France.

(4) IFREMER, Laboratoire ECOHAL, BP 21105, 44311 NANTES Cedex, France.

\section{RÉSUMÉ}

La technique de fluoromarquage des otolithes a permis d'évaluer à grande échelle les pratiques d'alevinage de truite (Salmo trutta L.) à des stades précoces (540 000 570000 alevins/an, la plupart vésiculés) pour trois cohortes (1994-95-96) dans une partie du Doubs longue de $40 \mathrm{~km}$ et fractionnée en trois zones (amont, moyenne et aval) par des aménagements hydroélectriques.

Il y a une relativement forte contribution (23 à $60 \%$ selon les cohortes et les zones) des alevins de repeuplement à la population de $0+$ en place en début automne. Le marquage de la totalité des alevins déversés a permis de montrer que les dynamiques de contribution des deux composantes (recrutement naturel et repeuplement) diffèrent. En effet, la contribution de l'alevinage s'érode au cours du temps au niveau de la population en place en zone courante peu profonde $(<1,2 \mathrm{~m})$. La baisse du taux de marqués a été forte entre les stades 1+ et 2+ pour la cohorte 1994 et elle a eu lieu plus précocement, entre les stades $0+$ et $1+$, pour la cohorte 1995. Sur les zones moyenne et aval, au delà du stade $0+$, la contribution du recrutement naturel est toujours largement dominante dans la population en place.

L'étude dans la pêche de loisir a mis en évidence un plus fort taux de truites marquées parmi les truites capturées par la pêche de loisir comparativement aux truites de la même cohorte échantillonnées l'année antérieure par pêche électrique en zone courante dans la population automnale en place. Le taux de marquées parmi les truites capturées jeunes, à 2+, est relativement élevé (12,5\% à $45,8 \%$ selon la zone). Cependant le taux de marqués diminue fortement avec l'âge dans la pêche de loisir et, globalement, la majorité des truites prélevées par la pêche de loisir dans le Doubs franco-suisse reste issue du recrutement naturel.

Mots-clés : Salmo trutta, repeuplement, marquage, otolithes, pêche. 


\title{
TEMPORAL DYNAMIC OF THE CONTRIBUTION OF STOCKING WITH PRECOCIOUS STAGES OF BROWN TROUT (SALMO TRUTTA L.) IN THE STANDING POPULATION AND IN ANGLERS CATCHES IN THE FRENCH-SWISS PART OF THE RIVER DOUBS.
}

\begin{abstract}
The technique of otoliths fluoromarking has been used to evaluate at a large scale the practices of brown trout (Salmo trutta L.) stocking (540 000 - 570 000/year, most of them as sac fry) for three cohorts 1994-95-96 in a part of the River Doubs having a length of $40 \mathrm{~km}$ and fragmented in three zones (upstream, median, downstream) by three hydroelectric dams.
\end{abstract}

For each of the three cohorts, there is a rather high contribution (23 to $60 \%$ according to the zone) of the stocked fry to the automnal 0+ standing population. The mass marking of all the stocked fry allowed to show that the dynamics of the contribution of the two components of recruitment (natural or stocked) differed. Indeed, the contribution of stocking decreased with time when measured in the autumnal standing population in running parts with a depth inferior to $1.2 \mathrm{~m}$. The sharp decrease of the percentage of marked trout was high between stages 1+ and 2+ for the cohort 1994 and it was earlier, between $0+$ and 1+, for the 1995 cohort. In the median and downstream zones, after the stage $0_{+}$, the contribution of natural recruitment is always widely dominant in the autumnal standing population.

The study showed a higher contribution of stocking among the anglers catches than in samples of the same cohort realized in lotic water the year before in autumnal standing population. The rate of marked trout is rather high (12.5 to $45.8 \%$ according to the zone) among the catches of young $2+$ trout. However, the rate of marked fish decreased sharply with the age of angled trout and the main part of trout globally angled in the French-Swiss part of the River Doubs are issued from the natural recruitment.

Key-words : Salmo trutta, restocking, marking, otoliths, angling.

\section{INTRODUCTION}

Le Doubs franco-suisse est la portion du Doubs, longue de $40 \mathrm{~km}$ et large de 13 à $40 \mathrm{~m}$, située entre Villers-le-Lac (Saut du Doubs) et son entrée en Suisse. La succession sur cette zone de trois ouvrages hydroélectriques entraîne d'importantes altérations du milieu (SRAE, 1986 ; DIREN Franche-Comté, 1994 ; BOUVIER, 1989, 1997). Le fonctionnement de ces barrages induit en effet des variations brutales du débit et donc des surfaces en eau. Ces impacts se combinent avec celui de l'accroissement de la charge en nutriments dont la manifestation la plus sensible consiste en de massives proliférations d'algues (DIREN Franche-Comté, op. cit.). Pour tenter de compenser l'impact des altérations du milieu sur la population de truite (Salmo trutta L.) et pour répondre à l'accroissement de la demande des pêcheurs, les différentes associations de pêche françaises et suisses pratiquent depuis plusieurs décennies des repeuplements avec des stades précoces qui n'avaient jamais été évalués en liaison avec le manque de techniques de marquage de masse de ces stades autorisant une étude à grande échelle.

Une collaboration entre le CSP et la Station INRA de Thonon ainsi que le développement de techniques de fluoromarquage des otolithes (voir revue par CHAMPIGNEULLE et ROJAS BELTRAN, 2001), ont permis de lever les obstacles (grande échelle et quantité importante d'alevins de petite taille déversés) inhérents à ce type 
d'étude. Des campagnes de fluoromarquage ont été menées en vue d'évaluer les dynamiques de contribution des deux sources de recrutement (repeuplement et recrutement naturel). II ne s'agissait pas d'un protocole visant à comparer divers modes de repeuplement (origines, stades, densités...). II s'agissait d'évaluer, cohorte par cohorte, la contribution des repeuplements tels qu'ils étaient pratiqués au cours de la période 19941996, en prenant en compte la fragmentation du Doubs franco-suisse par les ouvrages hydroélectriques et surtout en suivant l'évolution de leur contribution dans le temps, à la fois dans la population en place et dans la pêche de loisir. Le suivi de plusieurs zones et cohortes, bien qu'alourdissant le travail, offrait la possibilité de fournir des répétitions dans l'étude réalisée.

\section{SITE D'ÉTUDE}

Le Doubs franco-suisse est fragmenté en trois grandes zones (amont, moyenne et aval) par trois barrages (Figure 1). Le Tableau I indique les dimensions des surfaces de retenue et de courants des zones inter-barrages. La zone aval possède une surface de secteurs courants nettement plus importante que les deux autres (Tableau I). Les zones courantes comprennent de l'amont vers l'aval : un barrage en limite amont, une portion court-circuitée par une conduite forcée, une zone de restitution du débit turbiné plus ou moins soumise aux effets des éclusées. Les données hydrauliques et hydrologiques du Doubs franco-suisse sont présentées en détail par BOUVIER $(1989,1997)$ et la DIREN Franche-Comté (1994). Le début de l'année 1995 a été caractérisé par une forte hydrologie avec, pour chacun des trois premiers mois, un débit moyen mensuel de 1,6 à 2,0 fois supérieur au module (DEGORGI et CHAMPIGNEULLE, 2000). Sur le site d'étude, hormis le cas d'un petit affluent-frayère situé en limite aval du site, la fraie de la truite se déroule presque exclusivement sur le cours principal.

\section{Tableau I}

Dimensions des retenues et des zones courantes et densités de repeuplement (individus $/ 100 \mathrm{~m}^{2}$ ) pour les différents stades de déversement dans la période 1994-96 sur les zones du Doubs franco-suisse. L : longueur ; S : surface.

\section{Table I}

Dimensions of pools and runs and stocking densities (individuals/100 $\mathrm{m}^{2}$ ) at the different stocking stages in the period 1994-96 in the three zones of the FrenchSwiss part of the River Doubs. L: length; S: surface.

\begin{tabular}{|c|c|c|c|c|c|c|c|c|c|c|}
\hline ZONE & $\begin{array}{c}\mathrm{L} \\
(\mathrm{m})\end{array}$ & $\underset{\left(m^{2}\right)}{S}$ & $\begin{array}{c}1994 \\
\text { vésiculés } \\
\left(\mathrm{n} / 100 \mathrm{~m}^{2}\right)\end{array}$ & $\begin{array}{c}1994 \\
\text { truitelles } \\
\left(\mathrm{n} / 100 \mathrm{~m}^{2}\right)\end{array}$ & $\begin{array}{c}1995 \\
\text { vésiculés } \\
\left(\mathrm{n} / 100 \mathrm{~m}^{2}\right)\end{array}$ & $\begin{array}{c}1995 \\
\text { démarrés } \\
\left(\mathrm{n} / 100 \mathrm{~m}^{2}\right)\end{array}$ & $\begin{array}{c}1995 \\
\text { truitelles } \\
\left(\mathrm{n} / 100 \mathrm{~m}^{2}\right)\end{array}$ & $\begin{array}{c}1996 \\
\text { vésiculés } \\
\left(\mathrm{n} / 100 \mathrm{~m}^{2}\right)\end{array}$ & $\begin{array}{c}1996 \\
\text { démarrés } \\
\left(\mathrm{n} / 100 \mathrm{~m}^{2}\right)\end{array}$ & $\begin{array}{c}1996 \\
\text { truitelles } \\
\left(\mathrm{n} / 100 \mathrm{~m}^{2}\right)\end{array}$ \\
\hline \multicolumn{11}{|l|}{ AMONT } \\
\hline Retenue & 2700 & & 0 & 0 & 0 & 0 & 0 & 0 & 0 & 0 \\
\hline Courants & 12800 & 275700 & 18 & 8 & 0 & 7 & 9 & 0 & 4 & 9 \\
\hline \multicolumn{11}{|l|}{ MOYEN } \\
\hline Retenue & 1000 & & 0 & 0 & 0 & 0 & 0 & 0 & 0 & 0 \\
\hline Courants & 7500 & 186200 & 32 & 0 & 56 & 0 & 0 & 0 & 0 & 0 \\
\hline \multicolumn{11}{|l|}{ AVAL } \\
\hline Courants & 15500 & 629600 & 70 & 0 & 62 & 0 & 0 & 83 & 0 & 0 \\
\hline
\end{tabular}




\section{DOUBS FRANCO-SUISSE}

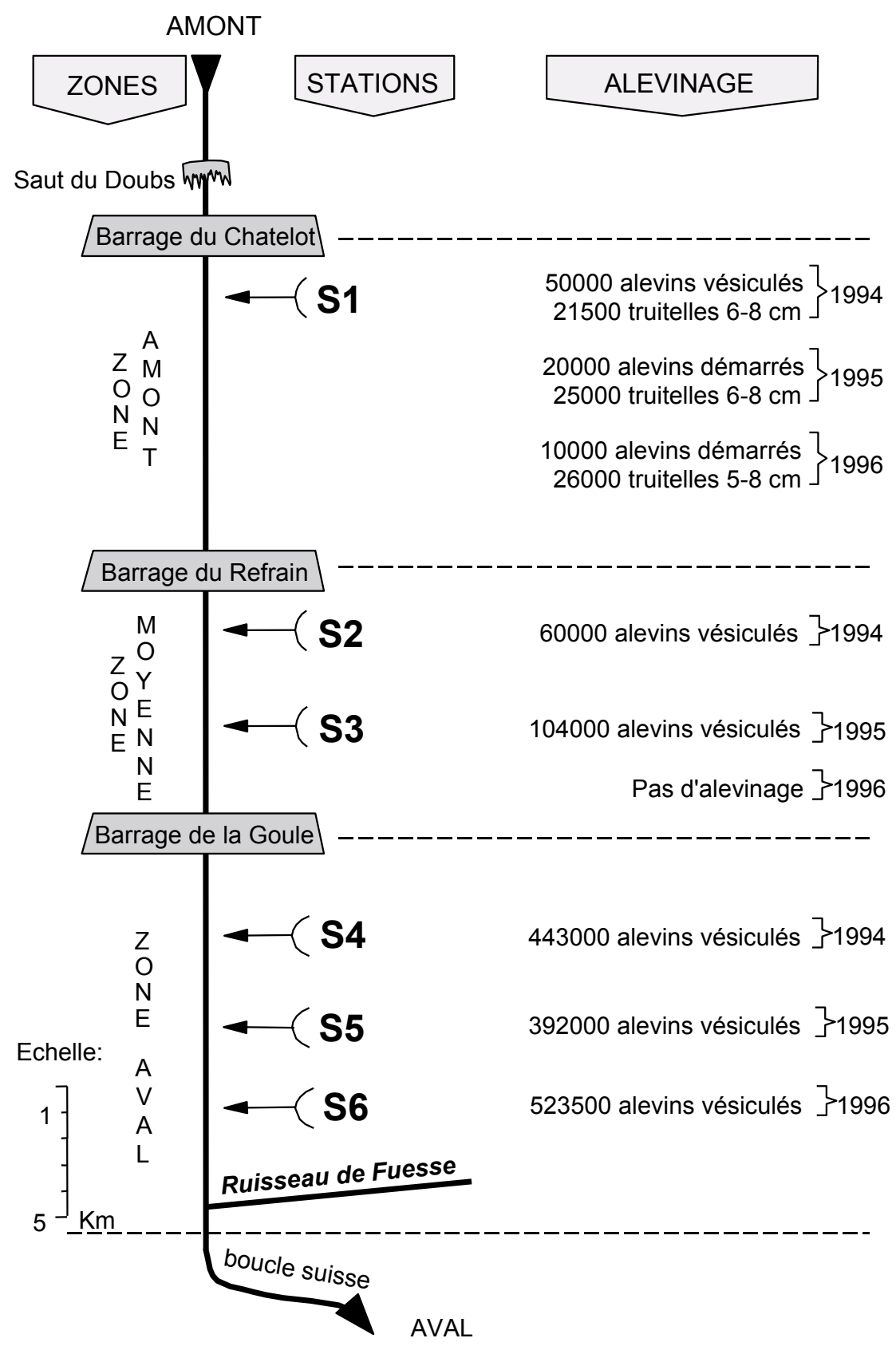

Figure 1

Doubs franco suisse. Localisation des barrages, des zones d'étude et des stations d'échantillonnage de la population en place. Pour chaque zone, le nombre et le stade des alevins déversés en zone lotique sont indiqués cohorte par cohorte (1994-95-96).

Figure 1

French-Swiss River Doubs: location of dams, zones under study and sampling stations for autumnal standing population. For each zone, the number and the developmental stage of the fry stocked in running water are given zone by zone for each cohort (1994-95-96). 


\section{MATÉRIEL ET MÉTHODES}

\section{Pratiques de repeuplement}

Une étude génétique (LARGIADER et SCHOLL, 1996) a montré que la truite autochtone du Doubs appartient au rameau évolutif méditerranéen. Or la plupart des souches de repeuplement utilisées dans le Doubs franco-suisse jusque dans les années 90 étaient originaires de stocks domestiques de forme atlantique, même si une part limitée provenait de croisements entre des géniteurs atlantiques domestiques et des truites sauvages, le plus souvent mâles.

La pression annuelle d'alevinage sur l'ensemble du Doubs franco-suisse est élevée, voisine de 550000 et sa répartition par zone et stade de déversement est indiquée dans la Figure 1. Les alevins destinés à l'alevinage d'une zone inter-barrage donnée ont été régulièrement répartis, par débit moyen ou faible, le long des berges des parties courantes peu profondes de l'ensemble de la zone concernée (TRIBOULET, comm. pers.). Les repeuplements sont pratiqués dans le Doubs franco-suisse depuis plusieurs dizaines d'années. II y a eu des hétérogénéités d'alevinage inter-zones (Tableau I) pour la période 1994-96. Les zones moyenne et aval ont été essentiellement repeuplées avec des stades précoces : alevins en fin de résorption de vésicule non nourris ou juste nourris quelques jours pour permettre de différer le relâcher en cas de conditions hydrologiques défavorables. Sur la zone aval, la densité d'alevinage a peu varié avec une valeur de 70-62-83 alevins vésiculés $/ 100 \mathrm{~m}^{2}$ de zone courante, respectivement pour chacune des années 1994-95-96. Pour la zone moyenne, comparativement à la zone aval, la densité d'alevinage a été inférieure (32 alevins vésiculés $/ 100 \mathrm{~m}^{2}$ ) en 1994, voisine (56 alevins vésiculés $/ 100 \mathrm{~m}^{2}$ ) en 1995 et il n'y a pas eu d'alevinage en 1996. A l'inverse des deux autres zones, ce sont surtout des stades plus avancés (alevins démarrés et truitelles de 5 à $8 \mathrm{~cm}$ ) qui ont été déversés dans la zone amont (Figure 1 et Tableau I).

\section{Marquage}

Tous les alevins de truite déversés dans le Doubs entre le barrage du Châtelot en amont et la limite amont de la boucle suisse du Doubs en aval (Figure 1) ont été marqués par fluoromarquage des otolithes lors des années 1994-95-96 (y compris les stades plus avancés déversés sur la zone amont). Le marquage a été réalisé à un stade très précoce (surtout alevins vésiculés, à l'exception d'une partie marquée au stade œuf en fin de période d'incubation en 1994). Le marquage a été effectué par balnéation dans une solution salée (5\%) contenant $1 \%$ de chlorhydrate de tétracycline. Le temps de balnéation a été de 10 à 20 min au stade œuf en fin d'incubation et de 3 min (pour le premier lot baigné dans la solution) à $3,5 \mathrm{~min}$ (pour les lots baignés ultérieurement dans la même solution) au stade d'alevins vésiculés. Deux lots témoins de quelques centaines d'alevins marqués en 1994 ont été gardés en pisciculture, puis examinés un peu après marquage et plus tardivement en fin de quatrième et de cinquième année (taille de 45 à $55 \mathrm{~cm}$ ). Les contrôles de marquage réalisés sur ces lots témoins indiquent $100 \%$ de rétention des marques.

La technique fluoromarquage des otolithes utilisée a été adaptée pour les salmonidés à gros œufs (ROJAS BELTRAN et al., 1995 ; CHAMPIGNEULLE et ROJAS BELTRAN, 2001) à partir de la technique utilisée sur les civelles par ALCOBENDAS et al. (1991). Elle rend possible le suivi d'alevinages avec des stades précoces. Comme il s'agit d'une méthode de marquage de masse, elle a permis d'identifier tous les alevins déversés dans le Doubs franco-suisse.

Compte tenu des caractéristiques des barrages (DIREN, 1994) et de ce que l'on connaît des comportements migratoires des truites en rivière aux différents âges 
(CRESSWELL, 1981 ; RAGON et al., 1990 ; MEYERS et al., 1992 ; OVIDIO, 1999), on peut faire l'hypothèse que la population en place en début automne et les captures par pêche sur le Doubs franco-suisse sont peu influençables par les apports en truites issues des parties situées en amont et en aval du Doubs franco-suisse. On peut alors adopter les équivalences : truite marquée $=$ truite issue du repeuplement et truite non marquée $=$ truite issue du recrutement naturel. niveaux :

Le suivi de la contribution des alevins marqués déversés a été réalisé à deux automne,

1) dans la population en place échantillonnée par pêche électrique en début

2) dans la fraction exploitée par la pêche récréative pratiquée sur le Doubs francosuisse.

\section{Population automnale en place}

La population en place a été échantillonnée en zone courante peu profonde $(<1,2 \mathrm{~m})$ sur 6 stations (S1 à S6) de grande taille $\left(1400\right.$ à $\left.6000 \mathrm{~m}^{2}\right)$ dont la localisation est indiquée en Figure 1. Deux types de milieux n'ont pas pu être prospectés en pêche électrique à pied : les retenues et les zones courantes profondes $(>1,2 \mathrm{~m})$.

Les même stations de référence ont fait l'objet de pêches électriques en octobre 94 , 95 et 96 permettant de respectivement recueillir un échantillon de $0+, 0+-1+, 0+-1+-2+$ dont l'âge et donc la cohorte d'appartenance ont été évalués par lecture d'écailles ou otolithométrie. Les déterminations d'âge ont cependant révélé que seul un faible nombre de truites 2+ a effectivement été échantillonné en 1996 (cohorte 94).

\section{Captures dans la pêche}

Le suivi a été réalisé du milieu de la saison de pêche 1997 jusqu'à la fin de la saison de pêche 1998. Le suivi des marqués dans la pêche a surtout été focalisé sur les zones moyenne et aval car elles ont le même mode de repeuplement et de gestion ainsi que la même réglementation. Un suivi additionnel limité a néanmoins été réalisé au cours de la seule saison 1998 dans la zone amont.

Le suivi de la contribution des truites marquées et non marquées capturées dans la pêche a été réalisé à partir d'échantillons récoltés selon le même protocole par des pêcheurs volontaires et par un garde échantillonnant le panier de pêcheurs. Les informations ont été recueillies sur les truites capturées ayant la taille légale minimale de $28 \mathrm{~cm}$ (longueur totale). Leur tête a été conservée pour analyse des otolithes et des écailles ont été prélevées pour déterminer leur âge. La zone de capture a été systématiquement spécifiée.

\section{Prélèvement et analyse des otolithes}

Les 2 sagittae ont été prélevées et débarrassées des matières organiques résiduelles. Chaque otolithe a été collé séparément sur une lame de verre avec une thermocolle (Crystalbond 509 AREMCO) chauffée à $120^{\circ} \mathrm{C}$. Les otolithes ont été polis jusqu'à atteindre le nucleus. Ils ont ensuite été observés avec un microscope Zeiss Axiolab équipé d'une lampe à vapeur de mercure (HBO 50) pour l'épifluorescence et d'un jeu de filtres Zeiss $\mathrm{n}^{\circ} 9$ (excitation bleu 450-490, FT510, émission LP520). 


\section{Analyse des données}

Les taux de marqués ont été évalués par zone, cohorte par cohorte, tant pour la population en place (capture par pêche électrique) que pour la pêche de loisir. Pour la population en place, les échantillons des diverses stations d'une même zone ont été regroupés. De même, les truites échantillonnées dans la pêche de loisir ont été regroupées par zone.

Les tests statistiques utilisés pour comparer les taux de marqués ont été le Chi deux et, dans le cas des petits échantillons, le test exact de Fisher (symbolisé TEF dans le texte).

\section{RÉSULTATS}

\section{Cohorte 1994 (Figure 2)}

Population en place $(0+, 1+, 2+)$

Le taux de $0+$ marqués est important et significativement (Chi deux, $\mathrm{P}<0,01$ ) plus élevé sur la zone amont $(60,0 \%)$ et la zone aval $(43,1 \%)$ que sur la zone moyenne $(27,9 \%)$. Sur les zones amont et moyenne, le taux de marqués ne diffère pas significativement entre les stades $0+$ et $1+$. Par contre, sur la zone aval, le taux de marqués présents au stade $1+(21,4 \%)$ est significativement inférieur (Chi deux, $\mathrm{P}<0,05)$ au taux de marqués présents au stade $0+(43,1 \%)$. Le taux de marqués baisse entre le stade $1+$ et le stade 2+ sur chacune des 3 zones, mais les pourcentages ne différent significativement $(T E F, P<0,05)$ que sur les zones amont et aval. On note en particulier sur cette dernière zone l'absence de truites $2+$ marquées.

Pêche de loisir $(3+, 4+)$

La contribution des marqués (cohorte 94) dans les captures par pêche des 3+ échantillonnés en milieu et fin de saison 1997 sur les zones moyenne et aval est identique $(22,2 \%)$. Pour la zone moyenne, ce taux n'est pas significativement différent (TEF, $\mathrm{P}>0,05)$ de celui observé au stade $2+$ dans la population en place sur la zone, mais les échantillons sont faibles (9 et 10). Pour la zone aval, zone principale de pêche, le taux de marqués parmi les $3+$ pêchés est, avec une valeur de 22,2 \%, significativement supérieur $(T E F, P<0,05)$ à celui $(0 \%, 0 / 27)$ observé parmi les $2+$ échantillonnés dans la population automnale en place.

Le taux de marqués est nul parmi les 4+ pêchés échantillonnés sur les zones amont et moyenne, cependant le nombre de captures examinées est faible (13 au total). Pour la zone aval, le taux de marqués parmi les 4+ pêchés est significativement $(T E F, P<0,05)$ plus faible $(3,2 \%)$ comparativement à celui de $22,2 \%$ observé au stade $3+$ dans la pêche.

\section{Cohorte 1995 (Figure 2)}

\section{Population en place $(0+, 1+)$}

Sur les trois zones, le taux de marqués est assez élevé au stade $0+$, variant entre 37,7 et $53,3 \%$, puis il s'effondre au stade $1+$ dans la population en place. Pour chacune des trois zones, le taux de marqués parmi les truites échantillonnées au stade 1+ est significativement inférieur (Chi deux, $\mathrm{P}<0,01)$ au taux de marqués observé chez les truites échantillonnées au stade $0+$. Pour les zones amont, moyenne et aval, les rapports : taux de 1+marqués/taux de $0+$ marqués sont respectivement $22,0 / 53,3 ; 10,5 / 37,7$ et $2,7 / 39,2$. 
Pêche de loisir (2+-3+)

Le taux de marqués parmi les 2+ pêchés échantillonnés sur la zone moyenne est de $27,3 \%$. Il est plus élevé, mais non statistiquement différent (TEF, $P>0,05$ ) du taux de marqués de 10,5\% observé parmi les 1+ échantillonnés dans la population automnale en place sur la zone moyenne.

Le taux de marqués parmi les 2+ pêchés échantillonnés sur la zone aval est élevé $(45,8 \%)$. II est très significativement supérieur (TEF, $\mathrm{P}<0,001)$ au taux de marqués $(2,7 \%)$ observé parmi les $1+$ échantillonnés dans la population en place sur la zone aval.

Le taux de marqués parmi les $3+$ pêchés échantillonnés sur les zones amont et moyenne sont faibles avec respectivement 9,7 et $5,3 \%$. Le taux de $5,3 \%$ de $3+$ marqués observé sur la zone moyenne est en forte baisse comparativement à celui $(27,3 \%)$ observé parmi les $2+$ pêchés.

Le taux de marqués parmi les $3+$ pêchés échantillonnés sur la zone aval est moyen, avec une valeur de $21,2 \%$. II est cependant significativement (Chi deux, $P<0,01$ ) inférieur au taux élevé de marqués $(45,8 \%)$ observé parmi les $2+$ pêchés échantillonnés sur la zone aval.

\section{Cohorte 1996 (Figure 2)}

$$
\text { Population en place }(0+)
$$

Le taux de 0+ marqués sur la zone amont est élevé $(42,1 \%)$ et significativement supérieur (Chi deux, $\mathrm{P}<0,01$ ) à celui de $22,9 \%$ observé sur la zone aval. Sur la zone aval, le taux de 0+ marqués observé en 1996 (22,9\%) est significativement (Chi deux, $\mathrm{P}<0,01)$ plus faible qu'en $1994(43,1 \%)$ et $1995(39,2 \%)$ malgré une densité d'alevinage supérieure en 1996 (83 alevins vésiculés $/ 100 \mathrm{~m}^{2}$ ) comparativement à 1994 (70 alevins vésiculés $/ 100 \mathrm{~m}^{2}$ ) et 1995 (62 alevins vésiculés $\left./ 100 \mathrm{~m}^{2}\right)$. Aucun des $1730+$ échantillonnés sur la zone moyenne non alevinée en 1996 n'était marqué.

Pêche de loisir (1+-2+)

La présence d'un petit nombre de $1+(3$, non représentés en Figure 2$)$ parmi les truites pêchées échantillonnées démontre l'existence d'individus à croissance très rapide entrant très précocement dans la pêche. II y avait parmi eux 2 marqués. Le taux de marqués parmi les 2+ pêchés échantillonnés en zone amont est de $12,5 \%$ mais l'échantillon est petit.

Malgré l'absence de déversement d'alevins précoces sur la zone moyenne pour la cohorte 1996 et l'absence probable de colonisation précoce à partir des zones adjacentes (suggérée par l'absence de 0+ marqués parmi les $1730+$ examinés dans la population en place, cf. ci-dessus), il y a un taux relativement élevé $(25,0 \%)$ de marqués parmi les 2+ pêchés sur la zone moyenne. Cependant l'échantillon examiné est faible (20). Ces marqués pourraient provenir de dévalaisons de la zone amont postérieurement au stade $0+$ en octobre.

Le taux de marqués parmi les 2+ pêchés échantillonnés sur la zone aval est élevé $(42,6 \%)$. Il est même significativement supérieur (Chi deux, $\mathrm{P}<0,01$ ) au taux de marqués $(22,9 \%)$ parmi les 0+ de la même cohorte échantillonnés en 1996 dans la population automnale en place sur la zone aval. 


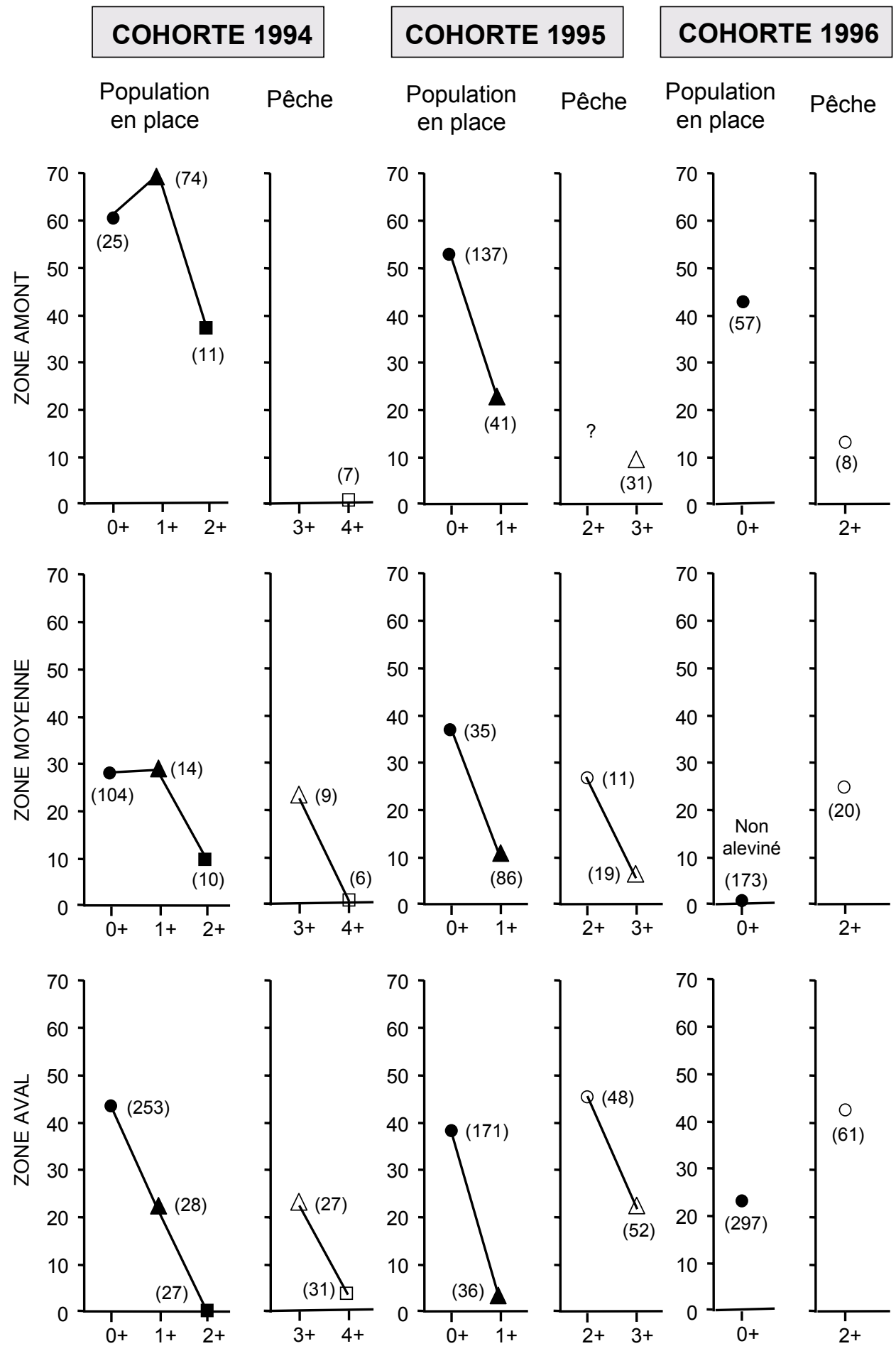

Figure 2

Evaluation, zone par zone et cohorte par cohorte, de la contribution (en \%) de l'alevinage sur le Doubs franco-suisse. (n) : taille de l'échantillon.

Figure 2

Percentage of marked fish among trout of different cohorts sampled in the three zones of the French-Swiss part of the river Doubs. $(n)$ : number of trout sampled. 


\section{DISCUSSION}

\section{Contribution du repeuplement au recrutement en $0+$ à l'automne}

Les campagnes de marquage ont montré qu'une partie non négligeable (23 à $43 \%$ selon les zones et cohortes) des $0+$ en place à l'automne sur les zones moyenne et aval du Doubs franco-suisse provient du repeuplement pratiqué avec des stades précoces.

Les taux de 0+ marqués les plus élevés (42 à $60 \%$ ) ont été observés sur la zone amont. Cette zone diffère des deux autres par le mode de repeuplement (déversement d'alevins de plus grande taille allant de 5 à $8 \mathrm{~cm}$ ) et parce qu'il s'agit de la zone subissant le plus directement les influences du barrage du Chatelot, le plus important des ouvrages hydroélectriques du Doubs franco-suisse. On ne peut dire lequel des deux facteurs est principalement en cause.

Dans le cas du Doubs franco-suisse, plusieurs études récentes (CSP, 1995 ; CSP, 1999 ; DEGORGI et CHAMPIGNEULLE, 2000) suggèrent une influence négative du régime hydraulique sur le recrutement naturel en juvéniles de truite. Les zones peu profondes abritant les stades précoces (œufs et alevins) sont particulièrement sensibles aux effets des variations de niveau fortes et/ou rapides. RIEGLER (1985) a mis en évidence sur la zone amont du Doubs franco-suisse des phénomènes d'exondation de frayères entraînant de fortes mortalités des œufs. Il est probable que, dans une rivière du type du Doubs franco-suisse, la période de post-émergence soit également une période très sensible. En effet, ELLIOTT (1994) a montré, pour la truite, l'existence d'une phase critique de fortes mortalités juste après l'émergence, lorsque les alevins commencent à se nourrir. HVIDSTEN (1985) a montré qu'une baisse rapide (50 cm en 30 minutes) provoquait de fortes pertes d'alevins 0+ par piégeage et mise à sec, diminuant significativement le recrutement en truites dans la rivière Nidelva, une rivière norvégienne avec des ouvrages hydroélectriques.

Les éléments précédents suggèrent l'existence de fortes mortalités aux stades précoces. Le protocole de l'étude ne permet cependant pas de savoir si le repeuplement s'est uniquement rajouté ou s'est en partie substitué au recrutement naturel. II existe en effet des phénomènes de régulation densité-dépendants survenant aux premiers stades. LE CREN (1961) a montré qu'un accroissement de la densité en alevins vésiculés de truite au delà d'un certain seuil conduisait à un plafonnement dans la densité de survivants à cinq mois. Dans un ruisseau anglais, ELLIOTT (1994) a même montré l'existence d'une baisse du recrutement en $0+$ de truite aux plus fortes densités en œufs naturellement déposés. MCMENEMY (1995) a montré, dans une grande rivière du Vermont repeuplée avec des alevins vésiculés de saumon atlantique, que des mises en charge de 2050 alevins vésiculés/100 $\mathrm{m}^{2}$ (moy. : 32) ont produit des densités égales ou supérieures de juvéniles $0+$ ou de 1 an comparativement à des mises en charges de 50-215 alevins vésiculés/100 $\mathrm{m}^{2}$ (moy. : 117).

Les densités d'alevinage en alevins vésiculés de 32 à $83 / 100 \mathrm{~m}^{2}$ pratiquées sur les parties lotiques des zones moyenne et aval du Doubs franco-suisse apparaissent plutôt faibles à moyennes comparativement aux valeurs indiquées dans la littérature pour les radiers et plats courants (CSP, 1999). Cependant, il est difficile de juger des chiffres de mise en charge dans le Doubs franco-suisse car ne sont connus, pour les stades précoces concernés, ni la capacité d'accueil des milieux repeuplés, ni à fortiori leur degré de saturation par le recrutement naturel au moment où l'alevinage est pratiqué. Par ailleurs, l'habitat du Doubs franco-suisse n'est pas suffisamment caractérisé et quantifié pour utiliser les données de GRANT et KRAMER (1990) et GRANT et al. (1998) qui montrent que la relation taille des territoires-taille des salmonidés permet de prédire la densité limite des salmonidés en rivière peu profondes et d'évaluer un indice de saturation de la capacité d'accueil. Des études récentes (GRANT et al., 1998 ; KEELEY, 2000) ont élargi l'approche 
en montrant que la taille des territoires variait en raison inverse de l'abondance en nourriture.

La plupart des études sur le déterminisme du recrutement précoce en alevins de truites ont porté sur de petites rivières ou des affluents frayères en débit naturel. Dans la présente étude, il s'agit du cours principal d'une grande rivière (largeur moyenne de $34 \mathrm{~m}$ sur la zone suivi sur le Doubs) avec des barrages hydroélectriques qui artificialisent le débit.

On ne peut donc pousser trop loin l'interprétation des taux de 0+ marqués observés en début automne. Ces taux constituent cependant un bon point de départ pour analyser l'évolution temporelle des deux composantes du recrutement en juvéniles. pêche

Dynamique des différentes fractions dans la population en place et dans la

L'étude a mis en évidence la dynamique suivante :

- la relativement forte contribution des alevins de repeuplement à la population de $0+$ en place en début automne en milieu courant s'érode au cours du temps au niveau de la population automnale en place dans ces mêmes parties courantes peu profondes $(<1,2 \mathrm{~m})$,

- par contre, il y a un plus fort taux de truites marquées dans les captures par la pêche de loisir au stade 2+ (cohorte 95) ou 3+ (cohorte 94), comparativement aux taux de marqués évalués antérieurement dans la population automnale en place dans les zones courantes aux stades 1+ (cohorte 95) ou 2+ (cohorte 94),

- pour les truites capturées par la pêche de loisir, le taux de marqués baisse avec l'âge à la capture.

Quelques hypothèses peuvent être avancées pour expliquer les variations temporelles observées:

a) II est possible que les juvéniles $0+$ et $1+$ issus du repeuplement, malgré leur relativement bonne implantation initiale, aient, aux stades plus avancés, des aptitudes de survie en milieu naturel inférieures à celles des alevins issus du recrutement naturel. La période d'hivernage, son début en particulier (SMITH et GRIFFITH, 1994), est en effet connue comme étant une phase critique. CUNJAK et POWER (1986) et CUNJAK (1988) ont montré, pour des juvéniles de saumon (Salmo salar L.), que le début de l'hiver est une période de stress et de mortalité accrue correspondant à l'acclimatation à des conditions environnementales rapidement changeantes. Les études réalisées par BACHMAN (1984) et DEVERILL et al. (1999) montrent qu'une des causes de la mortalité supérieure des truites de repeuplement issues de stocks domestiques est l'existence de dépenses énergétiques excessives.

b) Une autre hypothèse est l'existence d'une plus forte tendance à la dévalaison pour les truites issues du repeuplement. Plusieurs auteurs (SKROCHOWSKA, 1969 et CUINAT, 1971) indiquent que le comportement migrateur varie selon les souches et apparaît notablement plus développé chez les souches domestiquées, en particulier celles constituées à partir de truite de mer.

c) Des biais liés : 1) à un sous-échantillonnage des $1+$ et 2+ de plus grande taille dans la population en place qui pourrait avoir conduit à minimiser le taux de marqués restant présents à ces stades ; 2) à des différences dans la distribution spatio-temporelle aux différents âges pour les truites issues du repeuplement comparativement à celles issues du recrutement naturel. 
La pêche électrique est pratiquée uniquement en courants peu profonds $(<1,2 \mathrm{~m})$. Elle ne couvre pas tous les types d'habitats effectivement utilisés alors que la pêche à la ligne prospecte à la fois les zones courantes peu profondes et certains habitats plus profonds. JONES (1975) et CHAMPIGNEULLE (1978) ont montré que les truites plus âgées recherchent des milieux plus profonds. Une étude dans trois rivières norvégiennes (BREMSET et BERG, 1997) a montré l'existence de densités, de biomasses (en 1+ à 3+) et de tailles (à un âge donné) supérieures dans des mouilles comparativement aux zones courantes moins profondes.

d) des différences de capturabilité par la pêche de loisir.

Des différences de comportements et d'habitats ou microhabitats pourraient faciliter la capture des individus domestiques. MESA (1991) a montré en rivière artificielle pour des truites à gorge coupée de 15-20 cm (Oncorhynchus clarki) que les truites domestiques étaient plus agressives que les sauvages. VINCENT (1960) et MOYLE (1969) indiquent que des ombles de fontaines de souches domestiquées ont tendance à nager plus près de la surface que des ombles de première génération issus de parents sauvages.

Cette plus forte capturabilité peut être en partie liée à des aspects génétiques. En effet, une étude génétique a été réalisée (MEZZERA et LARGIADER, 2001) à la même période dans le Doubs 5 à $10 \mathrm{~km}$ en aval du Doubs franco-suisse. Cette étude a démontré une plus forte capturabilité par la pêche de loisir pour les génotypes atlantiques et hybrides comparativement aux truites méditerranéennes autochtones. Dans le cas du Doubs francosuisse, un tel phénomène pourrait en partie expliquer le taux de marqués plus élevé dans les captures par la pêche de loisir que le taux de marqués observé l'année antérieure dans la population en place.

La présente étude suggère que les truites issues du repeuplement rentrent plus rapidement dans la pêche (principalement au stade 2+) et en disparaissent plus rapidement que celles issues du recrutement naturel. Les échantillonnages de la population en place au stade $2+$ (cohorte 94$)$ en automne ont été réalisés après la phase d'exploitation maximale de la fraction repeuplée de la cohorte ; ceci pourrait alors contribuer à expliquer le faible taux de 2+ marqués observé dans la population automnale en place. Cet impact sélectif de la pêche suggère que la pêche de loisir pourrait contribuer à limiter la participation à la fraie des truites issues du repeuplement pratiqué sur le Doubs franco-suisse.

\section{CONCLUSION}

II n'y avait pas à notre connaissance de données publiées sur la contribution de repeuplements de truite au stade d'alevins vésiculés sur le cours principal de grandes rivières où existait par ailleurs une composante recrutement naturel. L'étude menée a démontré la faisabilité de ce type d'étude à grande échelle en associant des approches méthodologiques complémentaires : un fluoromarquage de masse, des pêches électriques pour échantillonner les populations en place et un suivi dans les captures par la pêche de loisir. II apparaît néanmoins utile de suggérer d'utiliser pour de futures études d'autres fluoromarqueurs que la chlorhydrate de tétracycline à cause du pouvoir antibiotique de ce dernier. CHAMPIGNEULLE et ROJAS BELTRAN (2001) ont testé avec succès l'alizarine RedS.

Le même type de dynamique temporelle de la contribution du repeuplement a récemment été observé (CAUDRON et CHAMPIGNEULLE, 2002) en zone amont du Fier, un torrent haut-savoyard repeuplé avec des alevins de 4 mois. II serait donc intéressant d'accroître ce type d'étude pour mieux évaluer le degré de généralisation des dynamiques observées. 
L'étude a montré que, malgré la relativement forte contribution initiale des repeuplements pratiqués, la part dominante de la population en place aux stades plus avancés et des captures dans la pêche de loisir reste issue du recrutement naturel. L'action prioritaire à mener est donc de renforcer le recrutement naturel en atténuant les facteurs de dégradation de la capacité d'accueil du milieu en particulier lors des phases les plus pénalisantes.

L'étude suggère que, chaque fois que possible, la dynamique des populations de truite faisant l'objet de repeuplements devrait être analysée en séparant les composantes recrutement naturel et repeuplement puisque ces deux composantes peuvent avoir des dynamiques différentes tant au niveau de la population en place que dans la fraction exploitée.

\section{REMERCIEMENTS}

De très nombreuses participations (administrations de la pêche françaises et suisses concernées, l'ensemble du personnel CSP impliqué, la DIREN et la DDAF, les Associations de pêcheurs, gardes-pêche, pêcheurs volontaires, pisciculteurs, bénévoles, étudiants, stagiaires) ont contribué à cette opération de grande envergure. Un remerciement particulier va à Mr TRIBOULET qui a eu un rôle moteur. Un hommage est rendu à Ricardo ROJAS BELTRAN qui a très fortement contribué à la première phase de l'étude et qui est malheureusement décédé en début juin 1997. Jacques ESCOMEL est remercié pour la réalisation des figures.

\section{BIBLIOGRAPHIE}

ALCOBENDAS M., LECOMTE F., CASTENET J., MEUNIER F.J., MAIRE P., HOLL M., 1991. Technique de marquage en masse des civelles (Anguilla anguilla) par balnéation rapide dans le fluorochrome. Application au marquage à la tétracycline de $500 \mathrm{~kg}$ de civelles. Bull. Fr. Pêche Piscic., 321, 43-54.

BACHMAN R.A., 1984. Foraging behavior of free-ranging wild and hatchery brown trout in stream. Trans. Amer. Fish. Soc., 113, 1-32.

BOUVIER J.C., 1989. Essai sur le régime hydrologique du Doubs. Actes de la Société jurassienne d'émulation. Office des eaux et de la protection de la nature du Canton du Jura, St-Ursanne, Suisse, $19 \mathrm{p}$.

BOUVIER J.C., 1997. Hydro-écologie. La boucle helvético-française du Doubs. Société d'Histoire Naturelle du Pays de Montbéliard, 167-186.

BREMSET G., BERG O.K., 1997. Density, size at age, and distribution of young Atlantic salmon (Salmo salar) and brown trout (Salmo trutta) in deep river pools. Can. J. Fish. Aquat. Sci., 54, 2827-2836.

CAUDRON A., CHAMPIGNEULLE A., 2002. Evolution temporelle de la contribution du repeuplement en truite (Salmo trutta L.) réalisée à des stades précoces dans le bassin amont du Fier (74). Bull. Fr. Pêche Piscic., 365/366, 455-469.

CHAMPIGNEULLE A., 1978. Caractéristiques de l'habitat piscicole et de la population de juvéniles sauvages de saumon atlantique (Salmo salar L.) sur le cours principal du Scorff (Morbihan). Thèse $3^{\text {ème }}$ cycle Biol. Anim. Fac. Sci. Univ. Rennes, $92 \mathrm{p}$.

CHAMPIGNEULLE A., ROJAS BELTRAN R., 2001. Le marquage des poissons. In : GERDEAUX (ed.), Gestion piscicole des grands plans d'eau, INRA, Paris, 311-346.

CRESSWELL R.C., 1981. Post-stocking movements and recapture of hatchery-reared trout released into flowing waters-a review. J. Fish. Biol., 18, 429-442. 
CSP, 1995. Etat de santé des populations salmonicoles et impacts des repeuplements sur le Doubs franco-helvétique. Rapport préliminaire: bilans des peuplements et résultats des marquages en 1994. Rapport CSP n 5-09-95, 1 vol., $34 \mathrm{p}$.

CSP, 1999. Gestion Piscicole. Interventions sur les populations de poissons. Repeuplement des cours d'eau salmonicoles. Collection mise au point, 1 vol., $256 \mathrm{p}$.

CUINAT R., 1971. Ecologie et repeuplement des cours d'eau à truites. Bull. Fr. Piscic., 240, 242, 243.

CUNJAK R.A., 1988. Physiological consequences of overwintering in streams: the cost of acclimatization? Can. J. Fish. Aquat. Sci., 45, 443-452.

CUNJAK R.A., POWER G., 1986. Winter habitat utilization by stream resident brook trout (Salvelinus fontinalis) and brown trout (Salmo trutta). Can. J. Fish. Aquat. Sci., 43, 1970-1981.

DEGIORGI F., CHAMPIGNEULLE A., 2000. Diagnose piscicole et mesure de l'efficacité des alevinages en truite sur le Doubs Franco-Helvétique. Rapport final Etude CSPINRA, $119 \mathrm{p}$.

DEVERILL J.I., ADAMS C.E., BEAN C.W., 1999. Prior residence, aggression and territory acquisition in hatchery-reared and wild brown trout. J. Fish Biol., 55, 868-875.

DIREN Franche-Comté, 1994. Le Doubs franco-helvétique. Aménagements hydroélectriques. Situations hydro-dynamiques et thermiques. Eléments de sédimentologie et de biologie. Région de Franche-Comté et communauté de travail du Jura ed., 90 p.

ELLIOTT J.M., 1994. Quantitative ecology and the brown trout. Oxford University press, 1 vol., $286 \mathrm{p}$.

GRANT J.W.A., KRAMER D.L., 1990. Territory size as a predictor of the upper limit to population density of juvenile salmonids in streams. Can. J. Fish. Aquat. Sci., 47, 1724-1737.

GRANT J.W.A., STEINGRIMSSON S.O., KEELEY E.R., CUNJAK R.A., 1998. Implications of territory size for the measurement and prediction of salmonid abundance in streams. Can. J. Fish. Aquat. Sci., 55 (suppl. 1), 181-190.

HVIDSTEN N.A., 1985. Mortality of presmolt Atlantic salmon (Salmo salar) and brown trout (Salmo trutta) caused by fluctuating water levels in the regulated River Nidelva, Central Norway. J. Fish Biol., 27, 711-718.

JONES A.N., 1975. A preliminary study of fish segregation in salmons spawning streams. J. Fish Biol., 7, 95-104.

KEELEY E.R., 2000. An experimental analysis of territory size in juvenile steelhead trout. Animal Behaviour, 59, 477-490.

LARGIADER C.R., SCHOLL A., 1996. Genetic introgression between native and introduced brown trout (Salmo trutta L.) populations in the Rhône River Basin. Molecular Ecology, 5, 417-426.

LE CREN E.D., 1961. How many fish survive? River Bds Ass., 57-64.

MCMENEMY J.R., 1995. Survival of Atlantic Salmon fry stocked at low density in the West River, Vermont. North Amer. J. Fish. Managmt., 15, 366-374.

MESA M.G., 1991. Variation in feeding, aggression, and position choice between hatchery and wild cutthroat trout in an artificial stream. Trans. Amer. Fish. Soc., 120, 723-727.

MEYERS L.S., THUEMLER T.F., KORNELY G.W., 1992. Seasonal movements of brown trout in Northeast Wisconsin. North Amer. J. Fish. Managmt., 12, 433-441.

MEZZERA M., LARGIADER C., 2001. Evidence for selective angling of introduced trout and their hybrids in a stocked brown trout population. J. Fish Biol., 59, 287-301.

MOYLE P.B., 1969. Comparative behaviour of young brook trout of domestic and wild origin. Prog. Fish Culturist, 31, 51-59.

OVIDIO M., 1999. Cycle annuel d'activité de la truite commune (Salmo trutta L.) adulte : étude par radiopistage dans un cours d'eau de l'Ardenne Belge. Bull. Fr. Pêche Piscic., 352, 1-18.

RAGON C., JONARD L., CUINAT R., 1990. Note technique. Etude, par piégeage et marquage, des montées de truites sur une rivière du Massif Central (La Faye) et un affluent, en 1986 et 1987. Bull. Fr. Pêche Piscic., 319, 47-49. 
RIEGLER K., 1985. Evaluation de l'importance de la fraye naturelle de la truite de rivière (Salmo trutta L.) dans les principales rivières du Canton de Neufchâtel. Diplôme de licence de sciences naturelles. Institut de zoologie de l'Université de Neuchâtel. 1 vol., $105 \mathrm{p}$.

ROJAS BELTRAN R., GILLET C., CHAMPIGNEULLE A., 1995. Immersion mass-marking of otoliths and bone tissues of embryos, yolk-sac fry and fingerlings of Arctic charr (Salvelinus alpinus L.). Nordic J. Freshw. Res., 71, 411-418.

SMITH R.W., GRIFFITH J.S., 1994. Survival of rainbow trout during their first winter in the Henrys fork of the Snake River, Idaho. Trans. Amer. Fish. Soc., 123, 747-756.

SKROCHOWSKA S., 1969. Migrations of the sea trout (Salmo trutta), brown trout, and their crosses. Pol. Arch. Hydrobiol., 16, 125-192.

SRAE, 1986. Aménagement hydroélectrique du Doubs franco-helvétique. Eléments de diagnose. 1 vol., $46 \mathrm{p}$.

VINCENT R.E., 1960. Some influences of domestication upon three stocks of brook trout (Salvelinus fontinalis). Trans. Amer. Fish. Soc., 89, 35-46. 
